ARTICLE

\title{
Anomalous electrical magnetochiral effect by chiral spin-cluster scattering
}

Hiroaki Ishizuka ${ }^{1 凶}$ \& Naoto Nagaosa ${ }^{1,2}$

The non-collinear spin configurations give rise to many nontrivial phenomena related to the Berry phase. They are often related to the vector and scalar spin chiralities. The scalar spin chirality leads to the topological Hall effect in metals, while the vector spin chirality to the ferroelectricity of spin origin, i.e., multiferroics in insulators. However, the role of the vector spin chirality in conducting systems has not yet been elucidated. Here we show theoretically that the spin correlation with vector spin chirality in chiral magnets scatters electrons asymmetrically, resulting in nonreciprocal transport phenomena, i.e., electrical magnetochiral effect (eMCE). This asymmetric scattering appears in the leading-order scattering term, implying a large nonreciprocity in the charge and spin currents. We find that the temperature and magnetic field dependence of the eMCE reproduces that observed in MnSi. Our results reveal the microscopic mechanism of eMCE and its potential in producing a large nonreciprocal response.

\footnotetext{
${ }^{1}$ Department of Applied Physics, The University of Tokyo, Bunkyo, Tokyo 113-8656, Japan. ${ }^{2}$ RIKEN Center for Emergent Matter Sciences (CEMS), Wako,

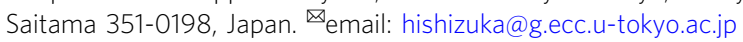


$\mathrm{N}$ on-coplanar magnetic configurations bring about rich electronic and magnetic properties of materials such as anomalous Hall effect $^{1-4}$, orbital magnetization ${ }^{5}$, and electric polarization in insulators ${ }^{6,7}$. These studies revealed that the vector $\chi_{i j}=\mathbf{S}_{i} \times \mathbf{S}_{j}$ and scalar $\chi_{i j k}=\left(\mathbf{S}_{i} \times \mathbf{S}_{j}\right) \cdot \mathbf{S}_{k}$ spin chiralities are central concepts in the physics of non-collinear spin structures. Since the spin operator is odd in $\mathcal{T}, \chi_{i j}$ is even while $\chi_{i j k}$ is odd. Therefore, $\chi_{i j k}$ is related to the magneto-transport; topological Hall effect associated with $\chi_{i j k}$, both intrinsic ${ }^{1,2}$ and extrinsic $^{8-10}$ mechanisms, are studied ${ }^{11}$. On the other hand, the inversion symmetry operation $P$ about the center of the bond connecting $i$ and $j$ reverses the sign of $\chi_{i j}$. The symmetry property implies $\chi_{i j}$ is related to the electric polarization of spin origin in insulators ${ }^{6,12}$. In conducting systems, on the other hand, the broken $P$ is subtle since the electric field in the metal is prohibited. However, there are several interesting nonreciprocal transport phenomena in noncentrosymmetric crystals ${ }^{13-15}$.

Nonreciprocal transport phenomena is a asymmetric dc electron transport, i.e., the current induced by a positive voltage is different from that by corresponding negative voltage. The reciprocal theorem by Onsager provides a basis to discuss the nonreciprocal linear responses ${ }^{16,17}$. This theorem originates from the time-reversal symmetry $\mathcal{T}$ of the microscopic dynamics, which is different from the macroscopic irreversibility. In transport theory, the Hermite symmetry also gives the reciprocal relation ${ }^{18}$, in addition to the space group symmetry of the crystal. Therefore, the breaking of $P$ alone does not necessarily lead to nonreciprocal responses. The nonreciprocity becomes even more subtle and rich for the nonlinear responses ${ }^{13,14}$. The nonreciprocal dc transport in solids manifests in the $I^{2}$ term of the $I-V$ curve, where $I$ is the injected electric current and $V$ is the voltage drop ${ }^{13,14}$. For example, the $I-V$ curve of eMCE follows ${ }^{13}$

$$
V=R_{0}(1+\gamma(B) I B) I,
$$

which means that the external magnetic field is needed to break $\mathcal{T}$ for this effect. Recent experiments found the nonreciprocal response in various nonmagnetic materials such as $\mathrm{Bi}$ helix ${ }^{13}$, semiconductors subject to gate potential ${ }^{14}$, molecular conductors $^{19}$, polar semiconductor ${ }^{20}$, and superconductor ${ }^{21}$. The nonreciprocal response also appears in magnetic materials, such as eMCE in metal/ferromagnet bilayer ${ }^{22}$, magnetic topological insulator $^{23}$, and chiral magnets ${ }^{24,25}$. In the magnetic systems, the magnetic ordering and fluctuation seem to play a crucial role in sharp contrast to the band structure effects dominating in the nonmagnetic systems. Among them, a recent paper reported a detailed experiment on the temperature and magnetic field dependence of eMCE in $\mathrm{MnSi}^{24}$, providing a useful set of information for theoretical studies. MnSi is a chiral magnet with a helical magnetic order in the zero field 26,27 . This material and its sister compounds are known for the magnetic-skyrmion crystal phase ${ }^{28-30}$. A recent experiment finds that $\mathrm{MnSi}$ also shows nonreciprocal response similar to the eMCE but with a nonmonotonic magnetic field dependence ${ }^{24}$; similar behavior also appears in $\mathrm{CrNb}_{3} \mathrm{~S}_{6}{ }^{25}$. These papers report a non-monotonic temperature dependence of the eMCE, which shows a maximum at around the magnetic transition temperature. The result implies the importance of magnetic fluctuation. However, the microscopic mechanism on how the magnetic fluctuation produces nonreciprocity remains elusive.

In this work, we theoretically study the nonreciprocal transport phenomena of electrons focusing on an asymmetric scattering by local magnetic correlation. We find the magnetic correlation in chiral magnets causes asymmetric scattering in the leading order of the scattering. The asymmetry produces a nonreciprocal response of electric current in nonmagnetic systems ${ }^{31}$. In contrast to Isobe et al. ${ }^{31}$, the magnetic scattering produces a larger nonreciprocal current because the asymmetric scattering appears in the leading order. Using the semiclassical Boltzmann theory, we show the magnitude of the nonreciprocal current is consistent with that in the experiment. Moreover, the temperature and magnetic field dependences reproduce the experiment. The consistency between the experiment and our theoretical results provides strong evidence of the extrinsic mechanism for nonreciprocal electric current.

\section{Results}

Asymmetric scattering rate. To study how the electron scattering produce nonreciprocal response, we here consider a model with itinerant electrons and localized spins coupled by exchange interaction. The Hamiltonian is

$$
H=\sum_{\mathbf{k} \sigma} \varepsilon_{\mathbf{k} \sigma} c_{\mathbf{k} \sigma}^{\dagger} c_{\mathbf{k} \sigma}+\frac{J}{N} \sum_{i, \mathbf{k}, \alpha, \beta} \gamma_{i \mathbf{k}} \mathbf{S}_{i} \cdot c_{\mathbf{k} \alpha}^{\dagger} \boldsymbol{\sigma}_{\alpha \beta} c_{\mathbf{k} \beta},
$$

where $c_{\mathbf{k} \sigma}\left(c_{\mathbf{k} \sigma}^{\dagger}\right)$ are respectively the annihilation (creation) operator of itinerant and localized electrons, $\sigma \equiv\left(\sigma^{x}, \sigma^{y}, \sigma^{z}\right)$ is the vector of Pauli matrices $\sigma^{a}(a=x, y, z), \varepsilon_{\mathbf{k} \sigma}=k^{2} /(2 m)-\sigma M-\mu$ is the eigenenergy of itinerant electrons with momentum $\mathbf{k}$ and spin $\sigma= \pm 1(+1$ for up spin and -1 for down spin), $k \equiv|\mathbf{k}|$, $\gamma_{i \mathbf{k}} \equiv e^{i \mathbf{k} \cdot \mathbf{r}_{i}}, J$ is the Kondo coupling between the localized spins and the itinerant electrons, and $\boldsymbol{S}_{i}$ is the localized moment at $\mathbf{r}_{i}$. Here, we assumed the magnetization is along the $z$ axis. This model is a classical- spin Kondo lattice model if the localized spins exists on every site, and is a Kondo impurity model if the spins exist only on a few sites $N_{s} \ll N$.

We calculate the scattering rate of electrons by the localized spins using Born approximation. In the first Born approximation, the scattering rate $W_{\mathbf{k} \sigma, \mathbf{k}^{\prime} \sigma^{\prime}}$ of electrons from the $\mathbf{k} \sigma$ state to the $\mathbf{k}^{\prime} \sigma^{\prime}$ state reads:

$$
W_{\mathbf{k} \sigma, \mathbf{k}^{\prime} \sigma^{\prime}}=\frac{2 \pi J^{2}}{N^{2}} \sum_{i, j, a, b} S_{i}^{a} S_{j}^{b} \sigma_{\sigma \sigma^{\prime}}^{a} \sigma_{\sigma^{\prime} \sigma}^{b} e^{\mathrm{i}\left(\mathbf{k}^{\prime}-\mathbf{k}\right) \cdot\left(\mathbf{r}_{i}-\mathbf{r}_{j}\right)} \delta\left(\varepsilon_{\mathbf{k} \sigma}-\varepsilon_{\mathbf{k}^{\prime} \sigma^{\prime}}\right) .
$$

Here we assume that the spins are classical and static, which is justified when the temperature is much higher than the typical energy of exchange interaction. The experimental situation in $\mathrm{MnSi}$ discussed below satisfies this condition. A recent work point outs that the asymmetry in the scattering rate $W_{\mathbf{k}, \mathbf{k}^{\prime}} \neq W_{-\mathbf{k},-\mathbf{k}^{\prime}}$ produces the nonreciprocity in the electron transport ${ }^{31}$ (Isobe et al. $^{31}$ considered spinless fermions.). Therefore, we focus on a similar asymmetry in $W_{\mathbf{k} \sigma, \mathbf{k}^{\prime} \sigma^{\prime}}$. The asymmetric part of the scattering rate $\left(W_{\mathbf{k} \sigma, \mathbf{k}^{\prime} \sigma^{\prime}}^{-} \equiv=\left(W_{\mathbf{k} \sigma, \mathbf{k}^{\prime} \sigma}-W_{-\mathbf{k} \sigma,-\mathbf{k}^{\prime} \sigma}\right) / 2\right)$ reads

$$
W_{\mathbf{k} \sigma, \mathbf{k}^{\prime} \sigma^{\prime}}^{-}=\frac{2 \pi J^{2}}{N^{2}} \sigma \delta_{\sigma, \bar{\sigma}^{\prime}} \sum_{i, j}^{N_{s}} \sin \left(\left(\mathbf{k}-\mathbf{k}^{\prime}\right) \cdot \mathbf{r}_{i j}\right)\left(\mathbf{S}_{i} \times \mathbf{S}_{j}\right)_{z} \delta\left(\varepsilon_{\mathbf{k} \sigma}-\varepsilon_{\mathbf{k}^{\prime} \sigma^{\prime}}\right) .
$$

Here, $\mathbf{r}_{i j}=\mathbf{r}_{i}-\mathbf{r}_{j}$ and $\bar{\sigma}=-\sigma$; we assumed $\varepsilon_{\mathbf{k} \sigma}=\varepsilon_{-\mathbf{k} \sigma}$. This asymmetric scattering vanishes when $N_{s}=1$; the sine function is always zero because $\mathbf{S}_{1} \times \mathbf{S}_{1}=\mathbf{0}$. Therefore, multiple spin scattering is necessary for the non-zero asymmetric scattering.

In the two spin case, the scattering rate reads

$$
W_{\mathbf{k} \sigma, \mathbf{k}^{\prime} \sigma^{\prime}}^{-}=\frac{4 \pi J^{2}}{N^{2}} \sigma \delta_{\sigma, \bar{\sigma}^{\prime}} \sin \left(\left(\mathbf{k}-\mathbf{k}^{\prime}\right) \cdot \mathbf{r}_{12}\right)\left(\mathbf{S}_{1} \times \mathbf{S}_{2}\right)_{z} \delta\left(\varepsilon_{\mathbf{k} \sigma}-\varepsilon_{\mathbf{k}^{\prime} \sigma^{\prime}}\right) .
$$

Hence, the asymmetry appears when a non-zero vector spin chirality exists, i.e., when the two spins are non-collinear. A previous work on multiferroics in insulators point outs the relation of the local spin current $\propto \mathbf{S}_{i} \times \mathbf{S}_{j}$ and electric polarization 6 . From a similar viewpoint, our result shows the local spin current scatters electrons asymmetrically depending on the spins (Fig. 1c). In addition, the result implies a finite 
a

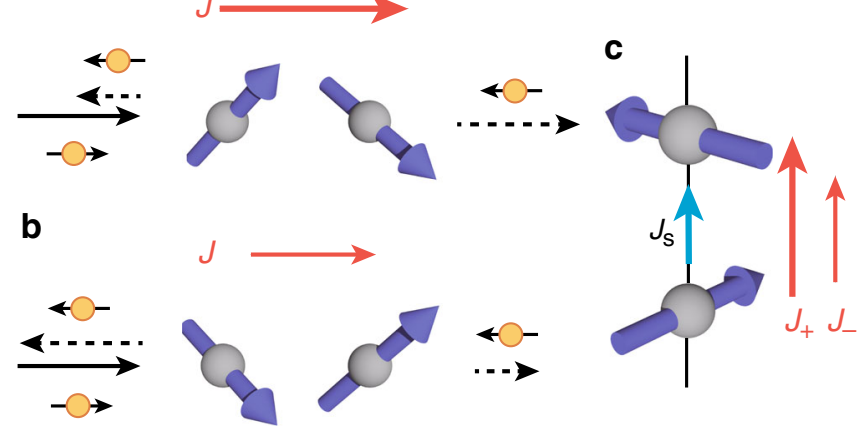

Fig. 1 Nonreciprocal transport by magnetic scattering. a, b Schematic figure of magnetic scattering by a two-spin cluster with finite vector spin chirality. The backward scattering by the two-spin spin cluster scatters incoming electron with up spin (the electron at the light bottom of the figure) depends on the vector spin chirality; less electrons are scattered backward in a compared to $\mathbf{b}$. The weaker backward scattering in a results in a larger current compared to $\mathbf{b}$. c Nonreciprocal spin current for $S^{z}$ in the paramagnetic case. The spin current of electrons $J_{s}$ flow along the direction of the supercurrent of magnetic moments because of the difference between the current for up-spin electrons and down-spin ones.

magnetization is necessary for the nonreciprocity because the asymmetric scattering rate in Eq. (5) has the opposite signs for $W_{\mathbf{k} \uparrow, \mathbf{k}^{\prime} \downarrow}^{-}$and $W_{\mathbf{k} \downarrow, \mathbf{k}^{\prime} \uparrow}^{-}$. Therefore, the asymmetry cancels when the itinerant electrons are paramagnetic $(M=0)$. In short, the above result implies nonreciprocity in the conductivity appears in a magnet when both the vector spin chirality and magetization are nonzero.

We note that $W_{\mathbf{k} \sigma, \mathbf{k}^{\prime} \sigma^{\prime}}^{-}$appears in the first Born approximation. This feature is in contrast to Isobe et al. ${ }^{31}$ where $W_{\mathbf{k} \sigma, \mathbf{k}^{\prime} \sigma^{\prime}}^{-}$appears from the second Born terms, i.e., higher-order in the perturbation. For the non-magnetic scatterers in time-reversal symmetric system, $W_{\mathbf{k} \sigma, \mathbf{k}^{\prime} \sigma^{\prime}}^{-}$is related to the skew scattering $W_{\mathbf{k}, \mathbf{k}^{\prime}}^{\mathrm{s}}=$ $\left(W_{\mathbf{k}, \mathbf{k}^{\prime}}-W_{\mathbf{k}^{\prime} \mathbf{k}}\right) / 2$ by $\mathcal{T}^{31}$. The skew scattering is prohibited in the first Born approximation because of the Hermiticity of the impurity potential. Therefore, the second-order term is the leading order. In contrast, the magnetic scattering considered here breaks $\mathcal{T}$. This difference of the symmetry allows non-zero $W_{\mathbf{k} \sigma, \mathbf{k}^{\prime} \sigma^{\prime}}^{-}$in the leading-order Born approximation. This result also implies that the magnetic scattering produces a larger nonreciprocal response.

In $\mathrm{MnSi}$, the spin-orbit interaction modifies the electronic bands, for instance, by a term such as $H_{\mathrm{SOI}}=\lambda \mathbf{k} \cdot \boldsymbol{\sigma}$. These terms, however, merely modifies our result by adding correction to the results above (see Supplementary Note 2). Therefore, we neglect the spin-orbit interaction on the itinerant electrons.

Boltzmann theory for nonreciprocal currents. To study how $W_{\mathbf{k} \sigma, \mathbf{k}^{\prime} \sigma^{\prime}}^{-}$contributes to the eMCE, we calculate the conductivity using the semiclassical Boltzmann theory. Using the relaxation time approximation, the Boltzmann equation reads

$$
e \mathbf{E} \cdot \nabla_{\mathbf{k}} f_{\mathbf{k} \sigma}=-\frac{f_{\mathbf{k} \sigma}-f_{\mathbf{k} \sigma}^{0}}{\tau}+\sum_{\mathbf{k}^{\prime}, \sigma^{\prime}} W_{\mathbf{k} \sigma, \mathbf{k}^{\prime} \sigma^{\prime}}^{-}\left(f_{\mathbf{k}^{\prime} \sigma^{\prime}}-f_{\mathbf{k} \sigma}\right) .
$$

Here, $e<0$ is the elementary charge, $\mathbf{E}=\left(E_{x}, E_{y}, E_{z}\right)$ is the applied electric field, and $f_{\mathbf{k} \sigma}$ is the electron density for the electrons with momentum $\mathbf{k}$ and spin $\sigma$. The relaxation time includes the contribution from non-magnetic scattering process as well as the symmetric scattering $W_{\mathbf{k} \sigma, \mathbf{k}^{\prime} \sigma^{\prime}}^{+} \equiv\left(W_{\mathbf{k} \sigma, \mathbf{k}^{\prime} \sigma^{\prime}}+W_{-\mathbf{k} \sigma,-\mathbf{k}^{\prime} \sigma^{\prime}}\right) / 2$ by the magnetic moments. For simplicity, we focus on the case $\mathbf{E}=$
$(0,0, E)$. In addition, we assume

$$
W_{\mathbf{k} \sigma, \mathbf{k}^{\prime} \sigma^{\prime}}^{-}=\left\{\begin{array}{lc}
0 & \text { if } \left.\sigma=\sigma^{\prime}\right) \\
2 \pi \sigma c\left(k_{z}-k_{z}^{\prime}\right) \delta\left(\varepsilon_{\mathbf{k} \sigma}-\varepsilon_{\mathbf{k}^{\prime} \sigma^{\prime}}\right) & \left(\text { if } \sigma \neq \sigma^{\prime}\right)
\end{array},\right.
$$

where $c=\frac{J^{2}}{N} \chi_{\mathrm{v}}$ is a real constant and $\chi_{\mathrm{v}} \equiv\left\langle\left(\mathbf{S}_{i} \times \mathbf{S}_{j}\right)_{z}\right\rangle$ is the thermal average of the $z$ component of the vector spin chirality between the nearest-neighbor spins along the $z$ axis. This asymmetric scattering term corresponds to the thermal average of the $k \ll 1$ case of the two-spin impurity cluster in Eq. (5).

We solve the Boltzmann equation in Eq. (6) with the scattering rate in Eq. (7) by expanding $f_{\mathbf{k} \sigma}$ up to the second order in $\mathbf{E}$ and linear order in $c^{31}$. Within this approximation, the nonreciprocal current reads

$$
J_{z}^{(2)}=-\frac{144 \pi}{5} \frac{\tau m}{e \mu^{2}} c M \sigma_{0}^{2} E^{2}
$$

where $2 \sigma_{0}=\frac{4 \mathrm{e}^{2} \tau \mu}{3 m n}$ is the linear conductivity of electrons at $M=0$ and $c=0$. Here, $n$ is the density of state at the Fermi level and we assumed $\mu \gg M$. Hence, the scattering by the two spins produce non-reciprocal current proportional to $c$ and magnetic polarization of the itinerant electrons $M$.

We also note that the two spin scattering produces the spin current. Using the same formalism, we find the spin current for $S^{z}$ reads

$$
J_{z}^{z}=-\frac{54 \pi \hbar}{5} \frac{\tau m}{\mathrm{e}^{2} \mu} c \sigma_{0}^{2} E^{2} .
$$

Unlike the charge current, the spin current appears without the spin polarization. Therefore, a paramagnet with the chiral spin correlation produces a finite spin current by simply flowing electric current. This spin current is qualitatively different from the spin current induced by the interfacial effect ${ }^{32}$, where the sign and magnitude of the spin current depends on the interface between the helical order and the leads. In contrast, the spin current in Eq. (9) is a phenomenon occuring in the bulk material.

Nonreciprocal charge current in chiral magnets. In the above mechanism, the nonreciprocal current depends on temperature and magnetic field via that of the magnetization and vector spin chirality. To investigate the dependence of nonlinear conductance, we here consider a classical ferromagnetic Heisenberg model on a cubic lattice with Dzyaloshinskii-Moriya interaction $27,30,33$,

$$
H_{\mathrm{cm}}=-J \sum_{\langle i, j\rangle} \mathbf{S}_{i} \cdot \mathbf{S}_{j}-\frac{D}{2} \sum_{\langle i, j\rangle} \mathbf{r}_{j i} \cdot \mathbf{S}_{i} \times \mathbf{S}_{j}-h \sum_{i} S_{i}^{z} .
$$

Here, the sum is over the nearest-neighbor bonds. Figure 2 shows the magnetic and transport properties of the above model; all results are obtained using Onsager's reaction field theory (See Methods section). Figure 2a is the plot of the magnetization to the magnetic field. The result shows a ferromagnetic magnetization curve below $T \lesssim 2 J$, which decreases monotonically with increasing temperature. In contrast, the vector spin chirality $\chi_{z}$ shows a non-monotonic temperature dependence. Figure $2 b$ shows the magnetic field dependence of $\left|\chi_{z}\right|$ for different $T$. When $T / J \lesssim 1$, the field-induced magnetization suppresses $\left|\chi_{z}\right| \rightarrow 0$ as $T \rightarrow 0$. With increasing temperature, the thermal fluctuation increase $\left|\chi_{z}\right|$ by suppressing the magnetization. The maximum is around $T / J \sim 2-3$ depending on $h$; the maximum tends to move to a higher $T$ as $h$ increases. Further increase of the temperature reduces the spin chirality because the thermal fluctuation dominates over the exchange interactions between the spins. 
a

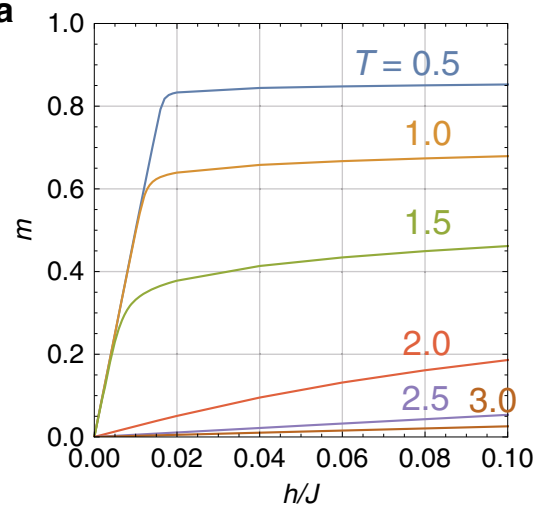

b

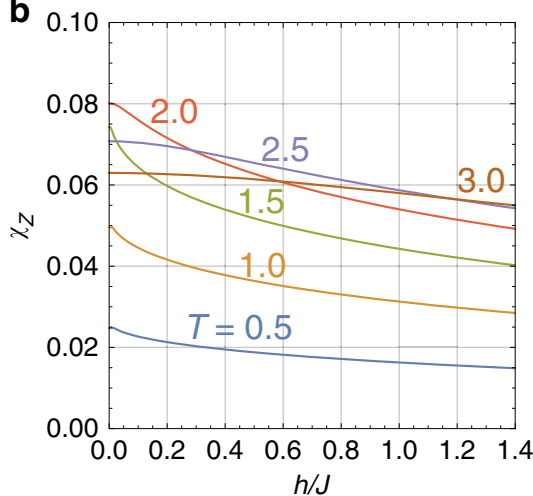

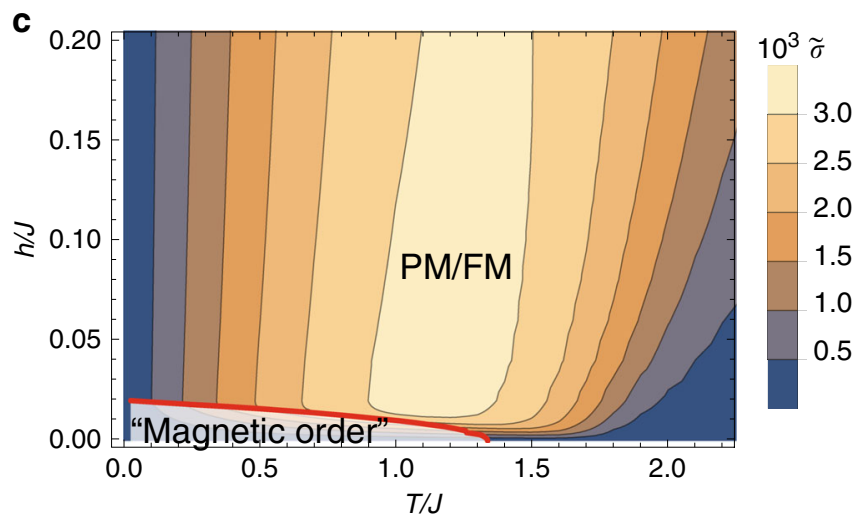

d

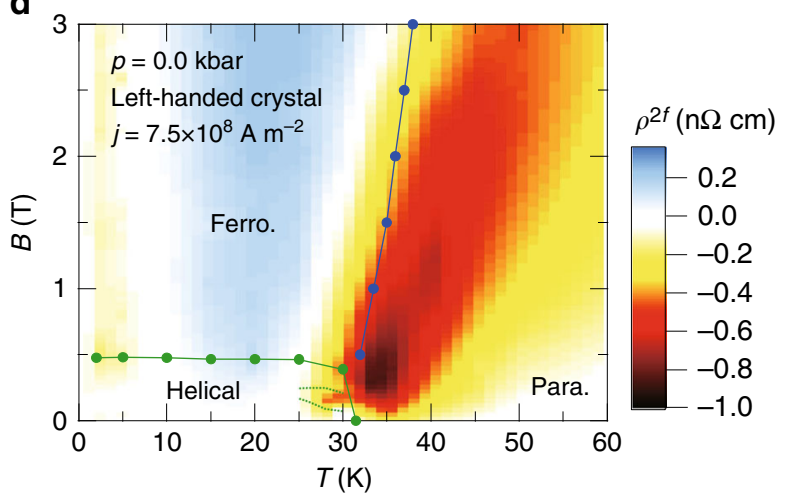

Fig. 2 Magnetic and transport properties of a three-dimensional chiral magnet. The magnetic-field dependence of $\mathbf{a}$ magnetization $M$ and $\mathbf{b}$ vector spin chirality $\chi_{z}$ for different temperature $T$. $\mathbf{c}$ is the contour plot of $\tilde{\sigma}=M \chi_{z}$. The results are for $D / J=0.2$. The red line is the phase boundary between the ordered and paramagnetic (PM/FM) phases, which is determined by $\lambda+D^{2} /(4 J)>-10^{-4}$. See Method section for details. $\mathbf{d}$ The contour plot of second harmonic resistivity $\rho^{2 f}\left(\propto \sigma^{(2)}\right)$. Reproduced from ref. ${ }^{24}$.

Equation (8) shows the nonreciprocal conductivity $\sigma^{(2)}$ is proportional to $\tilde{\sigma}^{(2)} \equiv M \chi_{z}$. Figure 2 c shows the contour plot of $\tilde{\sigma}^{(2)}$ in the $T-h$ plane. In the low temperature region, the result shows a small $\tilde{\sigma}^{(2)}$ owing to the suppression of the vector chirality. With increasing the temperature, $\tilde{\sigma}^{(2)}$ increases due to the increase of $\chi_{z}$ with a maximum around $T / J \sim 1.5-2 ; \tilde{\sigma}^{(2)}$ then decreases because both $M$ and $\chi_{z}$ is suppressed by the thermal fluctuation when $T / J \gg 1$. Figure $2 \mathrm{c}$ also shows the increase of the maximum with increasing the magnetic field. This is related to the increase of the maximum of $\chi_{z}$ discussed above. We find these behaviors remain robust even when we take into account the temperature dependence of the relaxation time $\tau$ (see Supplementary Note 1). They are also consistent with the experiment in $\mathrm{MnSi}$ as shown in Fig. $2 \mathrm{~d}^{24}$.

\section{Discussion}

To summarize, we studied the nonreciprocity of electric current produced by spin-cluster scattering. We find that the scattering process involving two spins cause an asymmetric scattering, which is proportional to the vector spin chirality. This effect appears at the leading order in the impurity scattering, i.e., within the first Born approximation. Therefore, we expect a large asymmetry in the scattering rate. Using the semiclassical Boltzmann theory, we find that this asymmetry produces nonreciprocal transport of electrons; $\sigma^{(2)}$ is proportional to the vector spin chirality and spin polarization of itinerant electrons. We also find the chiral-spin scattering produces nonreciprocal spin current, in contrast to recently-reported boundary effect ${ }^{32}$. As a consequence, $\sigma^{(2)}$ shows a non-monotonic temperature with a maximum around $T / J \sim 1$. This trend is consistent with the recent experiments in $\mathrm{MnSi}^{24}$ and $\mathrm{CrNb}_{3} \mathrm{~S}_{6}{ }^{25}$. In particular, the overall behavior of $\sigma^{(2)}$ well accounts for the eMCE in $\mathrm{MnSi}^{24}$.

The magnitude of the eMCE by the magnetic scattering is also consistent with the experiment in $\mathrm{MnSi}^{24}$. A recent experiment of $\mathrm{MnSi}$ finds the ratio of linear and nonreciprocal resistivities $\gamma(B)$ $I B \sim 10^{-4}-10^{-5}$ with $I=10^{9} \mathrm{Am}^{-2}$. When $\gamma(B) I B \ll 1$, the ratio reads $\gamma I B \sim-\sigma^{(2)} I / \sigma^{2}$. We estimate $\sigma^{(2)}$ using Eq. (8) assuming $J=10 \mathrm{meV}, D=1 \mathrm{meV}, a_{0}=4 \AA, m=9.109 \times 10^{-31} \mathrm{~kg}, \rho=$ $1 /\left(2 \mu_{\mathrm{F}} a_{0}^{3}\right) \sim 10^{39} \mathrm{~J}^{-1} \mathrm{~cm}^{-3}$, and $\tau=10^{-13}$ s. We use $\mu_{\mathrm{F}}=0.5 \mathrm{eV}$ and $M\left(=g \mu_{\mathrm{B}} H\right)=100 \mathrm{meV}$ because the bandwidth is $\sim 1 \mathrm{eV}^{34}$ and the spin polarization is in the order of $10 \%^{35}$. Using these values, we find $\sigma^{(2)} I / \sigma^{2} \sim 2 \times 10^{-5}$. Therefore, the result is roughly comparable with that observed in $\mathrm{MnSi}$.

The quantum effects of the spin such as Kondo effect on the spin cluster scattering are an interesting problem. Within the second Born approximation, the log singularity due to the second-Born approximation is absorbed in the renormalized exchange coupling $J$. Therefore, both the usual symmetric scattering and anti-symmetric scattering are enhanced (reduced) towards the Kondo temperature $T_{\mathrm{K}}$ for the antiferromagnetic (ferromagnetic) $J$. As the temperature is further lowered, the physics of multi-spin Kondo effect will show up and the nonreciprocal nature there is an important and interesting problem left for future studies.

\section{Methods}

Boltzmann theory. We used the semiclassical Boltzmann theory to calculate the nonreciprocal current. Assuming the steady state, the semiclassical Boltzmann equation reads

$$
e \mathbf{E} \cdot \nabla_{\mathbf{k}} f_{\mathbf{k} \sigma}=\sum_{\mathbf{k}^{\prime}, \sigma^{\prime}}\left(W_{\mathbf{k} \sigma, \mathbf{k}^{\prime} \sigma^{\prime}} f_{\mathbf{k}^{\prime} \sigma^{\prime}}-W_{\mathbf{k}^{\prime} \sigma^{\prime}, \mathbf{k} \sigma} f_{\mathbf{k} \sigma}\right) .
$$


Here, $e<0$ is the elementary charge, $\mathbf{E}=\left(E_{x}, E_{y}, E_{z}\right)$ is the applied electric field, and $f_{\mathbf{k} \sigma}$ is the electron density for the electrons with momentum $\mathbf{k}$ and spin $\sigma$. For simplicity, we focus on the case $\mathbf{E}=(0,0, E)$. The first (second) term in the righthand side of the equation represents the scattering of electrons from $\mathbf{k}^{\prime} \sigma^{\prime}(\mathbf{k} \sigma)$ to $\mathbf{k} \sigma$ $\left(\mathbf{k}^{\prime} \sigma^{\prime}\right)$. We approximate the symmetric part of the scattering rate by a relaxation time $\tau$. A similar approximation were used elsewhere to study transport phenomena related to a specific scattering term ${ }^{10,31,36,37}$. Within this approximation, the Boltzmann equation reads

$$
e \mathbf{E} \cdot \nabla_{\mathbf{k}} f_{\mathbf{k} \sigma}=-\frac{f_{\mathbf{k} \sigma}-f_{\mathbf{k} \sigma}^{0}}{\tau}+\sum_{\mathbf{k}^{\prime}, \sigma^{\prime}} W_{\mathbf{k} \sigma, \mathbf{k}^{\prime} \sigma^{\prime}}^{-}\left(f_{\mathbf{k}^{\prime} \sigma^{\prime}}-f_{\mathbf{k} \sigma}\right) .
$$

Here, we assume the form of asymmetric scattering rate to be

$$
W_{\mathbf{k} \sigma, \mathbf{k}^{\prime} \sigma^{\prime}}^{-}=\left\{\begin{array}{lc}
0 & \text { if } \left.\sigma=\sigma^{\prime}\right) \\
2 \pi \sigma c\left(k_{z}-k_{z}^{\prime}\right) \delta\left(\varepsilon_{\mathbf{k} \sigma}-\varepsilon_{\mathbf{k}^{\prime} \sigma^{\prime}}\right) & \left(\text { if } \sigma \neq \sigma^{\prime}\right)
\end{array}\right.
$$

where $c=\frac{J^{2}}{N} \chi_{\mathrm{v}}$ is a real constant and $\chi_{\mathrm{v}} \equiv\left\langle\left(\mathbf{S}_{i} \times \mathbf{S}_{j}\right)_{z}\right\rangle$ is the thermal average of the $z$ component of the vector spin chirality between the nearest-neighbor spins along the $z$ axis.

This asymmetric scattering term corresponds to the $k \ll 1$ case of the two-spin impurity cluster in Eq. (3). It also applies to the paramagnetic phase of the Kondo lattice models where the correlation length between the localized moments are short. In this case, the magnetic moments in Eq. (3) should be replaced by the thermal average,

$$
W_{\mathbf{k} \sigma, \mathbf{k}^{\prime} \bar{\sigma}}^{-}=\frac{2 \pi J^{2} \sigma}{N^{2}} \sum_{i, j}\left(\mathbf{k}-\mathbf{k}^{\prime}\right) \cdot \mathbf{r}_{i j}\left\langle\left(\mathbf{S}_{i} \times \mathbf{S}_{j}\right)_{z}\right\rangle \delta\left(\varepsilon_{\mathbf{k} \sigma}-\varepsilon_{\mathbf{k}^{\prime} \sigma^{\prime}}\right) .
$$

Here, the sum is over all localized moments in the system. This sum is reduced to the sum over nearest-neighbor bonds when the correlation length is similar or less than the lattice spacing, i.e., $\left\langle\left(\mathbf{S}_{i} \times \mathbf{S}_{j}\right)_{z}\right\rangle \sim 0$ for further-neighbor bonds. Assuming $\left\langle\left(\mathbf{S}_{i} \times \mathbf{S}_{j}\right)_{z}\right\rangle=\chi_{z} \neq 0$ only for the nearest-neighbor bonds along the $z$ axis, the constant $c$ reads $c=J^{2} \chi_{z}$ (we chose the unit of length as $\left|\mathbf{r}_{i j}\right|=1$ for the nearestneighbor bonds).

We solve the Boltzmann equation in Eq. (6) with the scattering rate in Eq. (7) by expanding $f_{\mathbf{k} \sigma}$ up to the second order in $\mathbf{E}$ and linear order in $c^{31} ; f_{\mathbf{k} \sigma}=$ $f_{\mathbf{k} \sigma}^{0}+\sum_{i=1,2, j=0,1} g_{\mathbf{k} \sigma}^{(i, j)}$ where $f_{\mathbf{k} \sigma}^{0}=1 /\left(1+e^{\beta \varepsilon_{\mathbf{k} \sigma}}\right)$ is the Fermi distribution function and $g_{\mathbf{k} \sigma}^{(i, j)}$ is the deviation from the equilibrium distribution in the $i$ th-order in $E$ and $j$ th order in $c$. We find

$$
\begin{gathered}
g_{\mathbf{k} \sigma}^{(1,0)}=-\tau e \mathbf{E} \cdot \nabla_{k} f_{\mathbf{k} \sigma}^{0}=\tau e \mathbf{E} \cdot \mathbf{v}_{\mathbf{k} \sigma} \delta\left(\varepsilon_{\mathbf{k} \sigma}\right), \\
g_{\mathbf{k} \sigma}^{(1,1)}=\tau \int \frac{d \mathbf{k}^{\prime}}{(2 \pi)^{3}} W_{\mathbf{k} \sigma, \mathbf{k}^{\prime} \sigma^{\prime}}^{-}\left(g_{\mathbf{k}^{\prime} \sigma^{\prime}}^{(1,0)}-g_{\mathbf{k} \sigma}^{(1,0)}\right), \\
g_{\mathbf{k} \sigma}^{(2,0)}=-\tau e \mathbf{E} \cdot \nabla_{\mathbf{k}} g_{\mathbf{k} \sigma}^{(1,0)}, \\
g_{\mathbf{k} \sigma}^{(2,1)}=-\tau e \mathbf{E} \cdot \nabla_{\mathbf{k}} g_{\mathbf{k} \sigma}^{(1,1)}+\tau \int \frac{d \mathbf{k}^{\prime}}{(2 \pi)^{3}} W_{\mathbf{k} \sigma, \mathbf{k}^{\prime} \sigma^{\prime}}^{-}\left(g_{\mathbf{k}^{\prime} \sigma^{\prime}}^{(2,0)}-g_{\mathbf{k} \sigma}^{(2,0)}\right) .
\end{gathered}
$$

In the Boltzmann theory, the current along the $z$ axis reads

$$
J_{z}=\mathrm{e} \sum_{\sigma} \int \frac{d \mathbf{k}}{(2 \pi)^{3}} v_{\mathbf{k} \sigma}^{z} f_{\mathbf{k} \sigma}=\mathrm{e} \sum_{\sigma} \sum_{i, j} \int \frac{d \mathbf{k}}{(2 \pi)^{3}} v_{\mathbf{k} \sigma}^{z} g_{\mathbf{k} \sigma}^{(i, j)} .
$$

Here, $\rho_{\sigma} \equiv \int \frac{d \mathbf{k}}{(2 \pi)^{3}} \delta\left(\varepsilon_{\mathbf{k} \sigma}\right)$ is the density of states for the electrons with spin $\sigma$. Therefore, the nonreciprocal current in $\mathcal{O}\left(E^{2}\right)$ reads

$$
J_{z}^{(2)}=\mathrm{e} \sum_{\sigma} \int \frac{d \mathbf{k}}{(2 \pi)^{3}} v_{\mathbf{k} \sigma}^{z}\left[g_{\mathbf{k} \sigma}^{(2,1)}+g_{\mathbf{k} \sigma}^{(2,2)}\right] .
$$

The $g_{\mathbf{k} \sigma}^{(2,1)}$ term contributes to the nonreciprocal current when the electronic band is asymmetric due to the absence of both time and spatial inversion symmetries ${ }^{20}$; this term vanishes in our case. Therefore, we here focus on the second term related to $g_{\mathbf{k} \sigma}^{(2,2)}$. The nonreciprocal current reads

$$
\begin{aligned}
& J_{z}^{(2)}=-\frac{16 \pi}{5} m \tau \mathrm{e} \rho_{+} \rho_{-} c M \frac{4 \mu^{2}-3 M^{2}}{\mu^{2}-M^{2}}\left(\frac{\tau \mathrm{e} E}{m}\right)^{2}, \\
& \sim-\frac{144 \pi}{5} \frac{\tau m}{\mathrm{e} \mu^{2}} c M \sigma_{0}^{2} E^{2} .
\end{aligned}
$$

Here, $\rho_{\sigma}=\frac{m}{2 \pi^{2}} \sqrt{2 m(\mu+\sigma M)}$ is the density of states for the electrons with spin $\sigma$. In the second line, we assumed $\mu \gg M$ and expanded up to the leading order in $M$; $2 \sigma_{0}=\frac{4 e^{2} \tau \mu}{3 m}$ is the linear conductivity of electrons at $M=0$ and $c=0$. Hence, the nonreciprocal current is proportional to the vector spin chirality $c=J^{2} \chi_{z}$ and magnetic polarization of the itinerant electrons $M$.
Similarly, the spin current reads

$$
\begin{gathered}
J_{z}^{(2)}=\frac{\hbar}{2} \sum_{\sigma} \int \frac{d \mathbf{k}}{(2 \pi)^{3}} \sigma v_{\mathbf{k} \sigma}^{z}\left[g_{\mathbf{k} \sigma}^{(2,1)}+g_{\mathbf{k} \sigma}^{(2,2)}\right], \\
=-\frac{4 \pi \hbar \tau^{3} \mathrm{e}^{2}}{m} c \rho_{+} \rho_{-} \mu\left\{1+\frac{1}{5}\left(\frac{\mu^{2}+3 M^{2}}{\mu^{2}-M^{2}}\right)\right\}, \\
\sim-\frac{54 \pi \hbar}{5} \frac{\tau m}{\mathrm{e}^{2} \mu} c \sigma_{0}^{2} E^{2} .
\end{gathered}
$$

The last equation is the result for $\mu \gg M$. The last equation implies the chiral correlation produces spin current in a paramagnetic phase without magnetization. A study on electric polarization by spin canting finds the polarization is parallel to $\mathbf{r}_{i j} \times \mathbf{j}_{\mathrm{s}}$ where $\mathbf{j}_{\mathrm{s}} \propto \mathbf{S}_{i} \times \mathbf{S}_{j}$ is the supercurrent of spin current ${ }^{6}$. In contrast, our result finds the component of $\mathbf{j}_{\mathrm{s}}$ parallel to $\mathbf{r}_{i j}$ is proportional to the spin current of electrons (Fig. 1a).

Magnetic phase diagram. Onsager's reaction field theory is used to calculate the magnetization and the vector spin chirality under external magnetic field ${ }^{38-40}$. This method incorporates the $\Sigma_{i}\left|\mathbf{S}_{i}\right|^{2}=N_{\mathrm{s}}$ constraint by introducing a Lagrange's multiplier $\lambda$. The effective Hamiltonian reads

$$
H_{\text {eff }}=\tilde{H}_{\mathrm{cm}}+\lambda \sum_{i}\left|\mathbf{S}_{i}\right|^{2} .
$$

Using this method, we find the magnetization and vector spin chirality are given by

$$
m_{z}=-\frac{h}{2 \lambda},
$$

and

$$
\chi_{z}=\frac{2 D T}{3 \pi^{2}} \int_{0}^{\Lambda} \frac{d q q^{4}}{\left(J q^{2}-\lambda\right)^{2}-D^{2} q^{2}} .
$$

Here, $\mathbf{q}=\left(q_{x}, q_{y}, q_{z}\right)$ is the wavenumber of the classical spin wave modes, $q=|\mathbf{q}|, \lambda$ is determined by

$$
1-\frac{h^{2}}{4 \lambda^{2}}=\int \frac{d \mathbf{q}}{(2 \pi)^{3}} T \operatorname{Tr}\left(\frac{1}{\lambda-J_{\mathbf{q}}}\right)
$$

and

$$
J_{\mathbf{q}}=\left(\begin{array}{ccc}
J q^{2} & \mathrm{i} D q_{z} & -\mathrm{i} D q_{y} \\
-\mathrm{i} D q_{z} & J q^{2} & \mathrm{i} D q_{x} \\
\mathrm{i} D q_{y} & -\mathrm{i} D q_{x} & J q^{2}
\end{array}\right) .
$$

This model does not show a phase transition for arbitrary choices of $h$ and $T$ when $D \neq 0$. This is an artifact of the approximation used in $J_{\boldsymbol{q}}$, where the model has a $S O(3)$ rotational symmetry in the momentum space. In the lattice model, however, the small anisotropy due to discrete rotational symmetry breaks the $S O(3)$ symmetry. To give an idea on the ordering by the anisotropy, we defined the system is magnetically ordered if $\lambda(T, h)+D^{2} /(4 J)<-10^{-4}$. Here, $-D^{2} /(4 J)$ is the ground state energy. In Fig. 2c, we plot the phase boundary by the red solid line.

\section{Data availability}

The authors declare that all data supporting the findings of this study are available within the paper.

Received: 15 September 2019; Accepted: 19 May 2020;

Published online: 12 June 2020

\section{References}

1. Ye, J. et al. Berry phase theory of the anomalous Hall effect: application to colossal magnetoresistance manganites. Phys. Rev. Lett. 83, 3737-3740 (1999).

2. Ohgushi, K., Murakami, S. \& Nagaosa, N. Spin anisotropy and quantum Hall effect in the kagomé lattice: chiral spin state based on a ferromagnet. Phys. Rev. B 62, R6065-R6068 (2000)

3. Taguchi, Y., Oohara, Y., Yoshizawa, H., Nagaosa, N. \& Tokura, Y. Spi chirality, Berry phase, and anomalous Hall effect in a frustrated ferromagnet. Science 291, 2573 (2001).

4. Neubauer, A. et al. Topological Hall effect in the A phase of MnSi. Phys. Rev. Lett. 102, 186602 (2009).

5. Shindou, R. \& Nagaosa, N. Orbital ferromagnetism and anomalous Hall effect in antiferromagnets on the distorted fcc lattice. Phys. Rev. Lett. 87, 116801 (2001).

6. Katsura, H., Nagaosa, N. \& Balatsky, A. V. Spin current and magnetoelectric effect in noncollinear magnets. Phys. Rev. Lett. 95, 057205 (2005). 
7. Bulaevskii, L. N., Batista, C. D., Mostovoy, M. V. \& Khomskii, D. I. Electronic orbital currents and polarization in Mott insulators. Phys. Rev. B 78, 024402 (2008).

8. Tatara, G. \& Kawamura, H. Chirality-driven anomalous Hall effect in weak coupling regime. J. Phys. Soc. Jpn 71, 2613-2616 (2002).

9. Denisov, K. S., Rozhansky, I. V., Averkiev, N. S. \& L'ahderanta, E. Electron scattering on a magnetic skyrmion in the nonadiabatic approximation. Phys. Rev. Lett. 117, 027202 (2016).

10. Ishizuka, H. \& Nagaosa, N. Spin chirality induced skew scattering and anomalous Hall effect in chiral magnets. Sci. Adv. 4, eaap9962 (2018).

11. Nagaosa, N., Sinova, J., Onoda, S., MacDonald, A. H. \& Ong, N. P. Anomalous Hall effect. Rev. Mod. Phys. 82, 1539-1592 (2010).

12. Tokura, Y., Seki, S. \& Nagaosa, N. Multiferroics of spin origin. Rep. Prog. Phys. 77, 076501 (2014).

13. Rikken, G. L. J. A., Folling, J. \& Wyder, P. Electrical magnetochiral anisotropy. Phys. Rev. Lett. 87, 236602 (2001).

14. Rikken, G. L. J. A. \& Wyder, P. Magnetoelectric anisotropy in diffusive transport. Phys. Rev. Lett. 94, 016601 (2005).

15. Tokura, Y. \& Nagaosa, N. Nonreciprocal responses from noncentrosymmetric quantum materials. Nat. Commun. 9, 3740 (2018).

16. Onsager, L. Reciprocal relations in irreversible process. I. Phys. Rev. 37, 405-426 (1931).

17. Kubo, R. Statistical-mechanical theory of irreversible process. I. General theory and simple applications to magnetic and conduction problems. J. Phys. Soc. Jpn 12, 570-586 (1957).

18. Fisher, D. S. \& Lee, P. A. Relation between conductivity and transmission matrix. Phys. Rev. B 23, 6851-6854 (1981).

19. Pop, F., Auban-Senzier, P., Canadell, E., Rikken, G. L. J. A. \& Avarvari, N. Electrical magnetochiral anisotropy in a bulk chiral molecular conductor. Nat. Commun. 5, 3757 (2014).

20. Ideue, T. et al. Bulk rectification effect in a polar semiconductor. Nat. Phys. 13, 578-583 (2017).

21. Wakatsuki, R. et al. Nonreciprocal charge transport in noncentrosymmetric superconductor. Sci. Adv. 3, e1602390 (2017).

22. Avci, A. O. et al. Unidirectional spin Hall magnetoresistance in ferromagnet/ normal metal bilayers. Nat. Phys. 11, 570-575 (2015).

23. Yasuda, K. et al. Large unidirectional magnetoresistance in a magnetic topological insulator. Phys. Rev. Lett. 117, 159903 (2019).

24. Yokouchi, T. et al. Electrical magnetochiral effect induced by chiral spin fluctuations. Nat. Commun. 8, 866 (2017).

25. Aoki, R., Kousaka, Y. \& Togawa, Y. Anomalous nonreciprocal electrical transport on chiral magnetic order. Phys. Rev. Lett. 122, 057206 (2019).

26. Ishikawa, Y., Tajima, K., Bloch, D. \& Roth, M. Helical spin structure in manganese silicide MnSi. Sol. Stat. Commun. 19, 525-528 (1976).

27. Bak, P. \& Jensen, M. H. Theory of helival magnetic structures and phase transition in MnSi and FeGe. J. Phys. C Sol. Stat. Phys. 13, L881-885 (1980).

28. Rößler, U. K., Bogdanov, A. N. \& Pfleiderer, C. Spontaneous skyrmion ground states in magnetic metals. Nature 442, 797-801 (2006).

29. Mühlbauer, S. et al. Skyrmion lattice in a chiral magnet. Science 323, 915-919 (2009).

30. Yu, X. Z. et al. Real-space observation of a two-dimensional skyrmion crystal. Nature 465, 901-904 (2010).

31. Isobe, H., $\mathrm{Xu}, \mathrm{S}$.-Y. \& Fu, L. High-frequency rectification via chiral Bloch electrons. Sci. Adv. 6, eaay2497 (2020).

32. Okumura, S., Ishizuka, H., Kato, Y., Ohe, J.-I. \& Motome, Y. Spin-current diode with a monoaxial chiral magnet. Appl. Phys. Lett. 115, 012401 (2019).

33. Landau, L. D., Lifshitz, E. M. \& Pitaevskii, L. P. Electrodynamics of Continuous Media. Vol. 8, p. 178-179 (Elsevier, 2008).

34. Jeong, T. \& Pickett, W. E. Implications of the B20 crystal structure for the magnetoelectronic structure of MnSi. Phys. Rev. B 70, 075114 (2004)
35. Lee, M., Onose, Y., Tokura, Y. \& Ong, N. P. Hidden constant in the anomalous Hall effect of high-purity magnet MnSi. Phys. Rev. B 75, 172403 (2007).

36. Ishizuka, H. \& Nagaosa, N. Noncommutative quantum mechanics and skew scattering in ferromganetic metals. Phys. Rev. B 96, 165202 (2017).

37. Ishizuka, H. \& Nagaosa, N. Impurity-induced spin chirality and skew scattering in magnetic metals. New J. Phys. 20, 123027 (2018).

38. Onsager, L. Electric moments of molecules in liquids. J. Am. Chem. Soc. 58, 1486-1493 (1936)

39. Brout, R. \& Thomas, H. Molecular field theory, the Onsager reaction field and the spherical model. Physics 3, 317-329 (1967).

40. Matsuura, M. et al. Classical and quantum spin dynamics in the fcc antiferromagnet $\mathrm{NiS}_{2}$ with frustration. Phys. Rev. B 68, 094409 (2003).

\section{Acknowledgements}

We thank T. Arima, Y. Fujishiro, N. Kanazawa, T. Morimoto, and Y. Tokura for fruitful discussions. This work was supported by JSPS KAKENHI Grant Numbers JP18H04222, JP18H03676, and JP19K14649, and JST CREST Grant Numbers JPMJCR16F1 and JPMJCR1874.

\section{Author contributions}

H.I. conducted the scattering rate analysis. H.I. and N.N. contributed equally to the calculation and the analysis of the Boltzmann theory. The manuscript was prepared by the two authors. N.N. supervised the project.

\section{Competing interests}

The authors declare no competing interests.

\section{Additional information}

Supplementary information is available for this paper at https://doi.org/10.1038/s41467 020-16751-2.

Correspondence and requests for materials should be addressed to H.I.

Peer review information Nature Communications thanks the anonymous reviewer(s) for their contribution to the peer review of this work.

Reprints and permission information is available at http://www.nature.com/reprints

Publisher's note Springer Nature remains neutral with regard to jurisdictional claims in published maps and institutional affiliations.

Open Access This article is licensed under a Creative Common Attribution 4.0 International License, which permits use, sharing, adaptation, distribution and reproduction in any medium or format, as long as you give appropriate credit to the original author(s) and the source, provide a link to the Creative Commons license, and indicate if changes were made. The images or other third party material in this article are included in the article's Creative Commons license, unless indicated otherwise in a credit line to the material. If material is not included in the article's Creative Commons license and your intended use is not permitted by statutory regulation or exceeds the permitted use, you will need to obtain permission directly from the copyright holder. To view a copy of this license, visit http://creativecommons.org/ licenses/by/4.0/

(C) The Author(s) 2020 